\title{
BCR-ABL Truncation due to Premature Translation Termination as a Mechanism of Resistance to Kinase Inhibitors
}

\author{
Wanlong $\mathrm{Ma}^{\mathrm{a}}$ Hagop Kantarjian ${ }^{\mathrm{b}}$ Chen-Hsiung Yeh ${ }^{\mathrm{a}}$ Zhong J. Zhang ${ }^{\mathrm{a}}$ \\ Jorge Cortes $^{\mathrm{b}}$ Maher Albitar $^{\mathrm{a}}$ \\ ${ }^{a}$ Department of Hematology and Oncology, Quest Diagnostics Nichols Institute, San Juan Capistrano, Calif., and \\ ${ }^{b}$ Department of Leukemia, M.D. Anderson Cancer Center, University of Texas, Houston, Tex., USA
}

The Philadelphia $(\mathrm{Ph})$ chromosome, a shortened version of chromosome 22, results from a reciprocal translocation between chromosomes $9 \mathrm{q} 34$ and 22q11 [1-3]. The $\mathrm{Ph}$ translocation positions the $\mathrm{c}-A B L$ gene of chromosome 9 downstream from the breakpoint cluster region $(B C R)$ on chromosome 22 ; the resulting fusion gene produces a 190 - or $210-\mathrm{kDa}$ hybrid protein with constitutive kinase activity associated with chronic myelogenous leukemia (CML). The impressive clinical efficacy of imatinib mesylate, a selective and effective ABL kinase inhibitor, has revolutionized the treatment of CML. However, the development of resistance to imatinib, which occurs over months to years, constitutes a major drawback in the treatment of advanced CML $[4,5]$. Mechanisms leading to drug resistance include amplification of the $B C R-A B L$ gene, acquired additional genomic alterations, and most importantly, specific mutations within the $A B L$ kinase domain that impede drug binding [2, 3, 6-8].

The ATP-binding site is usually formed between the two lobes of the tyrosine kinase domain. Because the ATP-binding motif is highly conserved, most tyrosine kinase inhibitors generated have been ATP mimetics. Imatinib and other newer agents, such as nilotinib and dasatinib, bind to the ATP-binding cleft within the activation loop (A-loop) of the ABL kinase, establishing extensive contacts with residues lining the cleft and blocking access of ATP to the cleft. Thus, subsequent tyrosine phosphor- ylation of the substrate is inhibited [5-9]. These inhibitors differ from one another in their molecular structure, how they bind to the BCR-ABL protein, and what other tyrosine kinases they target. These differences lead to different patterns of activity and resistance, resulting in distinct profiles of resistance mutations that are likely to evolve within the kinase domain.

Interrogation of the imatinib database indicates that 136 amino acid changes at 100 different ABL residues have been reported to date, and that the 16 most commonly mutated amino acids account for about $87 \%$ of all reported mutations; these include mutations at T315 (12.1\%), E255 (10.7\%), Y253 (9.3\%), M351 (9.2\%), and G250 (8.5\%) [9]. Other types of mutations, such as deletions and insertions, have only recently been described. Reported in-frame deletions in exon 4 of the ABL kinase include $\Delta 184-274$ and $\Delta 248-274$, both of which display a phenotype of inactive kinase, lack of growth factor independence, and increased sensitivity to imatinib, nilotinib, and dasatinib [10-12]. An in-frame deletion skipping the first half of exon 8 has also been documented [13]. In addition, a 35-nt insertion derived from intron 8 was found positioned between the junction of ABL exons 8 and 9 in a patient with chronic CML resistant to imatinib [14]. The resulting truncated BCR-ABL protein lacks the C-terminal nuclear localization, DNA binding, and actin-binding domains.

\section{KARGER}

Fax +4161306 1234 E-Mail karger@karger.ch www.karger.com
(C) 2009 S. Karger AG, Basel

0001-5792/09/1211-0027\$26.00/0

Accessible online at:

www.karger.com/aha
Maher Albitar

Quest Diagnostics Nichols Institute, 33608 Ortega Highway

San Juan Capistrano, CA 92675 (USA)

Tel. +1 949728 4784, Fax +1 9497287781

E-Mail maher.x.albitar@questdiagnostics.com 


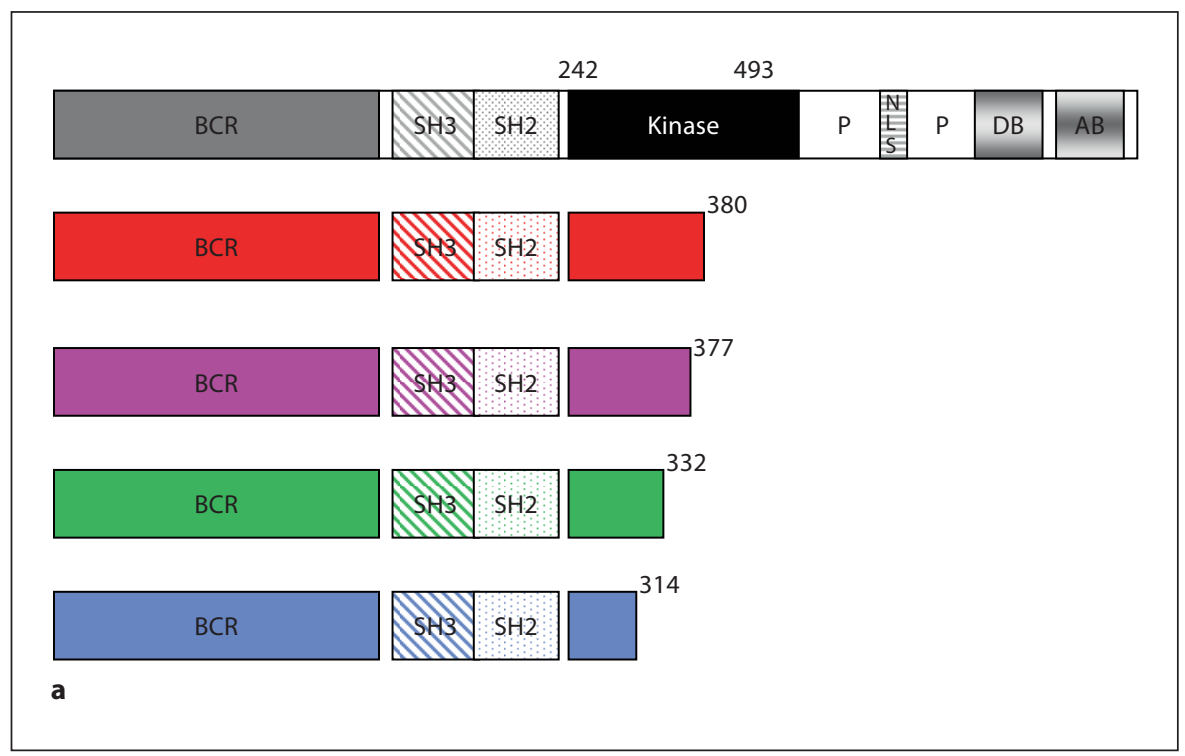

Fig. 1. Schematic diagram and sequence alignment of wild-type and four mutant $\mathrm{Bcr}-\mathrm{Abl}$ proteins resulting from premature termination mutations. a The organization of wild-type and four mutant protein domains is shown. b Amino acid sequences are aligned and labeled in black (wild type), red (exon 7 Del), pink (exon 7 2 -nt Del), green (exon $6997 \mathrm{C} \rightarrow \mathrm{T}$ ) and blue (exon 6 4-nt Ins). Sequence differences caused by frameshift mutations are underlined. Premature stop codons are indicated by asterisks, and the position of T315 is marked in yellow.
271

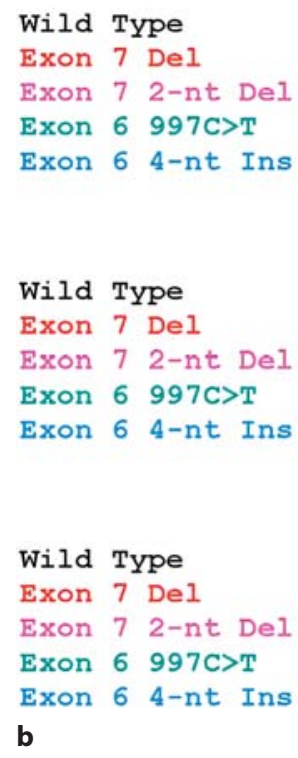

KTLKEDTMEVEEFLKEAAVMKEI KHPNLVQLLGVCTREPP

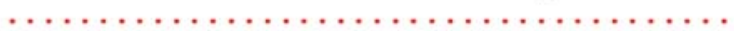

The key structures of the ABL kinase, part of the BCR$\mathrm{ABL}$ leukemogenic molecule, consist of $\mathrm{SH} 3, \mathrm{SH} 2$ and kinase domains, proline-rich regions $(\mathrm{P})$, as well as a nuclear localization signal (NLS), and DNA- and actin-binding (DB and $\mathrm{AB}$ ) sites (fig. 1a). The core kinase domain is organized into an N-lobe, which carries the highly conserved nucleotide-phosphate-binding site for ATP (the Ploop), and a large carboxyl-terminal C-lobe containing the flexible activation loop (the A-loop), a regulatory subunit for kinase activity $[13,15,16]$. Frequency studies of
BCR-ABL mutations detected in clinical CML samples revealed that mutations mainly cluster in four distinct regions of the kinase domain. Mutations in the P-loop (amino acids 244-255) are most common, followed by the T315I mutation, which causes global conformational changes. M351, the activation loop hinge, interacts with the $\mathrm{SH} 2$ domain and participates in autoregulation of kinase activity. The fourth cluster encompasses the A-loop from residues 381-402 [13]. Imatinib resistance is associated with at least 15 single amino acid mutations at 13 
Exon 7 deletion

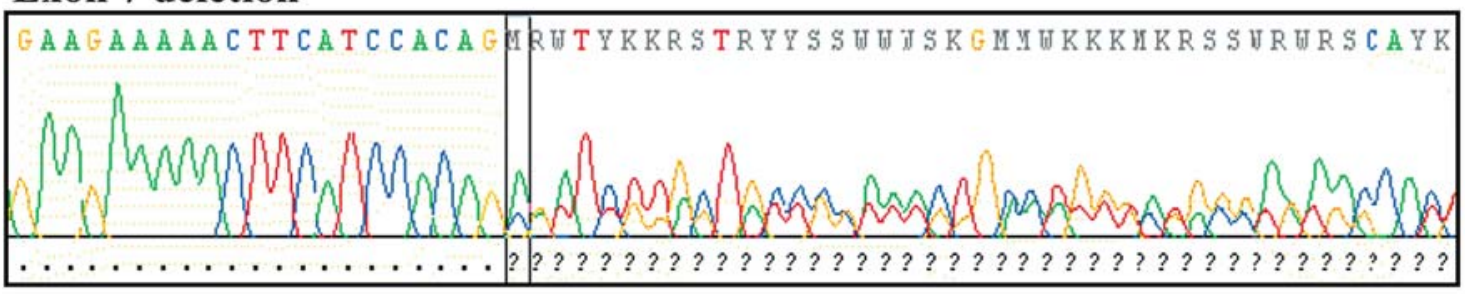

\section{Exon 6, 4-nt frameshift insertion}

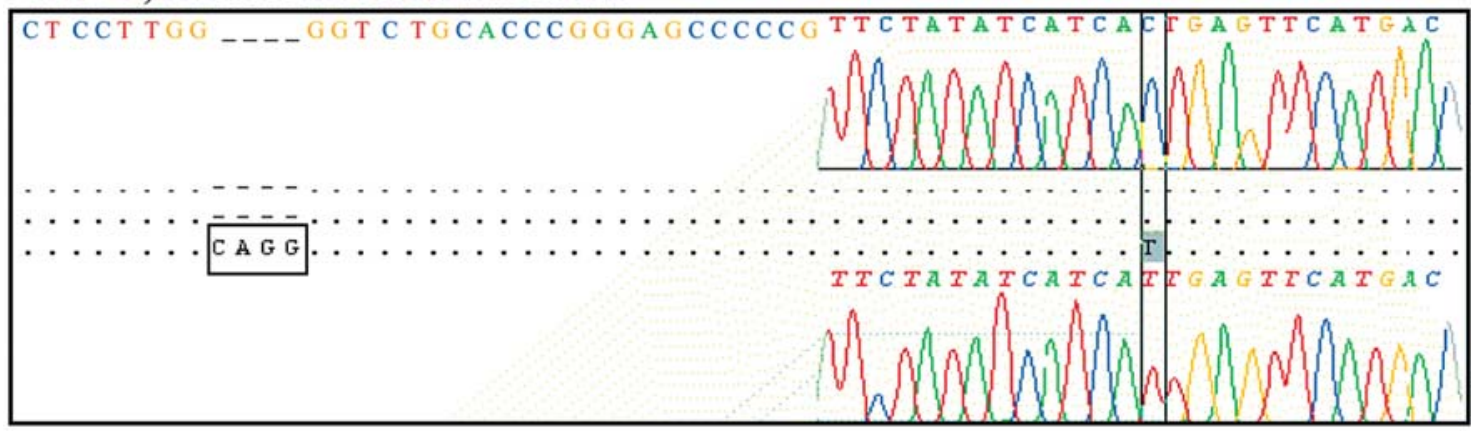

\section{Exon 6, 997C $>$ T point mutation}

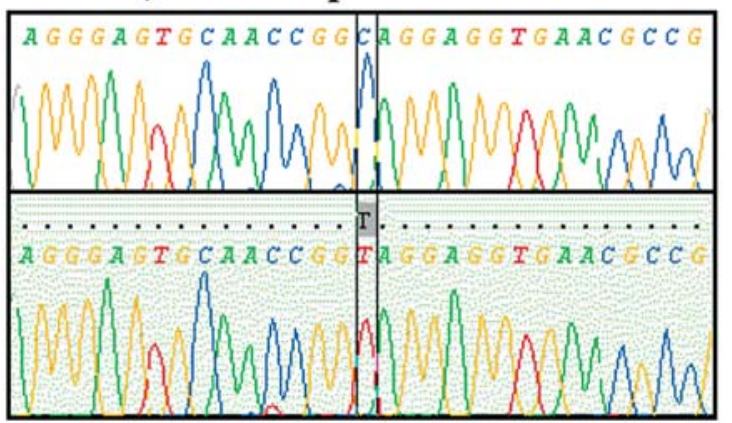

Exon 7, 2-nt deletion

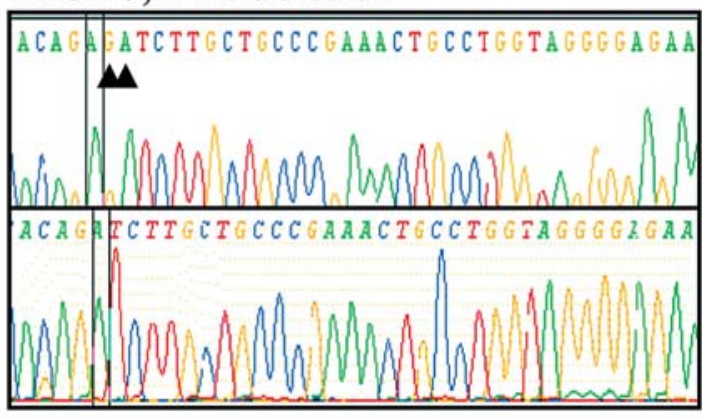

Fig. 2. Representative sequence traces of exon 6 and 7 frameshift or point mutations in the $a b l$ gene. The exon 7 deletion causes a frameshift and a premature termination codon in exon 8 . The bases deleted are indicated with question marks. For exon 6 frameshift, a CAGG tetranucleotide insertion at the junction of exons 5 and 6 is indicated by a rectangular box, and a $\mathrm{C} \rightarrow \mathrm{T}$ silent mutation is also highlighted. A $\mathrm{C} \rightarrow \mathrm{T}$ mutation at nucleotide 997 in exon 6 also results in a termination codon ( $\mathrm{cag} \rightarrow \operatorname{tag})$. In exon 7, 2-nt deletion is marked by arrows. The sequences are compared to an Abl kinase domain reference sequence using sequencing analysis software, which aligns the sequences and highlights single or multiple mutations.

distinct positions within the ABL kinase domain; the most frequently involved positions are T315 and E255, known to be crucial for drug binding $[2,13,17,18]$. P-loop as well as T315 mutations disrupt and shift the conformational equilibrium of the kinase to favor the active state and allosterically prevent imatinib and other kinase inhibitor binding. Accordingly, most of these point mutations confer resistance to imatinib, nilotinib, and dasatinib and are associated with a worse prognosis than are mutations elsewhere [16, 19].

BCR-ABL Truncation Mutations in Drug-Resistant Leukemia
Using a sensitive reverse transcription polymerase chain reaction and DNA sequencing approaches, four previously unrecognized ABL kinase mutations were found in CML and acute lymphoblastic leukemia (ALL) patients with resistance to imatinib, nilotinib, and/or dasatinib: an exon 7 deletion in 3 CML patients, a 4-nt insertion (908insCAGG) near the exon $5 / 6$ junction in 1 CML case, a 2-nt deletion near the end of exon 7 (10881089GA) in 1 CML patient, and an exon 6 point mutation $(997 \mathrm{C} \rightarrow \mathrm{T})$ in $1 \mathrm{ALL}$ patient. All of these mutations, al- 
though through different mechanisms (e.g. deletion, insertion or point mutation), create premature stop codons and cause termination at residues $381,315,378$, and 333 , respectively, leading to two mutants lacking the A-loop (exon 7 Del and exon 7 2-nt Del), one missing T315, M351 and the A-loop (exon 64 -nt Ins), and one terminated at codon 333 , in addition to the lack of the C-terminal region downstream of the kinase domain (fig. 1). These premature termination mutations, along with the previously documented 35-nt insertion in exon 8 (35Ins), may constitute a new class of mutations that (1) cause truncation of the BCR-ABL kinase; (2) abolish the regulatory element in the ABL kinase domain and the downstream C-terminal region, and (3) confer significant drug resistance. Consistent with the 35Ins mutant (terminated at residue 484) which has conformational alterations similar to that in T315I and with comparable drug resistance [14], the four new ABL kinase mutations, with a truncation pattern analogous to that seen in 35Ins, may also display the T315I-like resistance profile.

Although deletions in both the SH3 domain and the $\mathrm{C}$-terminal proline-rich regions have been shown to greatly impair BCR-ABL leukemogenesis in mice [20], deletion of the $\mathrm{ABL}$ actin-binding domain or the entire $\mathrm{C}$-terminal region downstream of the kinase domain induces CML-like myeloproliferative disorders in vivo [21]. In addition, the $\mathrm{N}$-terminal portion (encompassing amino acids 1-507) required for homomeric and heteromeric interactions among ABL kinase and its binding partners was found to play an important role in the modulation of kinase activity [22]. Therefore, the truncated proteins resulting from premature translational termination are expected to possess leukemogenic activity and to induce dramatic conformational changes to jeopardize drug binding and cause resistance. It is noteworthy that these newly identified ABL truncation mutations are structurally closely related to the SRC family kinases. The striking similarity between the catalytically active states of the ABL and SRC kinases suggests that these truncated, allosteric mutants could exert effects on drug binding resembling those of SRC kinase or be regulated in an SRC-like manner [23]. Indeed, SRC kinase inhibitors such as PP2, 4-amino-5-(4-chlorophenyl)-7-( $t$-butyl) pyrazolo(3,4-d)pyrimidine, and PD180970 have been found to effectively induce apoptosis in imatinib-resistant CML cells [24].

Alternative or aberrant splicing in $B C R-A B L$ transcripts has been observed in a significant proportion of CML patients and is increasingly recognized as a mechanism for drug resistance $[10,13,25,26]$. At least three
$B C R-A B L$ splice variants are potentially associated with drug resistance $[10,13,14]$. The deletion spanning the entire exon 7 reported here adds a new member to the list. Given that both normal and aberrantly spliced forms may be present, and many deletion mutations are interpreted as sequencing trace overlays and thus escape diagnosis, extra care should be taken not to disregard these as poorquality sequence traces (fig. 2). Another potential alternative splice variant frequently detected from imatinibresistant CML samples in our laboratory is 35Ins, in which a $35-n t$ insertion derived from intron 8 was found positioned between the junction of $A B L$ exons 8 and 9 . This insertion also causes a frameshift leading to a premature stop codon following 10 intron-encoded amino acids.

In summary, we describe several novel mutations that result in BCR-ABL truncations of various lengths within the kinase domain, leading to mutants missing the $\mathrm{ABL}$ C-terminal NLS, DB, and AB domains. These kinase domain truncations, in addition to the previously reported 35Ins, may emerge as a novel mutation category and a previously undiscovered mechanism associated with drug resistance. The four premature translation termination mutations detected in our study represent the first example linking this class of mutation to multiple drug resistance in CML and ALL patients.

References

$\mathrm{Ma} /$ Kantarjian/Yeh/Zhang/Cortes/ Albitar 
5 Druker BJ: STI571 (Gleevec ${ }^{\mathrm{TM}}$ ) as a paradigm for cancer therapy. Trends Mol Med 2002;8: S14-S18.

6 Kantarjian H, Giles F, Wunderle L, Bhalla K, O'Brien S, Wassmann B, Tanaka C, Manley P, Rae P, Mietlowski W, Bochinski K, Hochhaus A, Griffin JD, Hoelzer D, Albitar M, Dugan M, Cortes J, Alland L, Ottmann OG: Nilotinib in imatinib-resistant CML and Philadelphia chromosome-positive ALL. N Engl J Med 2006;354:2542-2551.

7 Talpaz M, Shah NP, Kantarjian H, Donato N, Nicoll J, Paquette R, Cortes J, O'Brien S, Nicaise C, Bleickardt E, Blackwood-Chirchir MA, Iyer V, Chen T-T, Huang F, Decillis AP, Sawyers CL: Dasatinib in imatinib-resistant Philadelphia chromosome-positive leukemias. N Engl J Med 2006;354:2531-2541.

8 Druker BJ: Circumventing resistance to kinase-inhibitor therapy. N Engl J Med 2006; 354:2594-2596.

-9 Hochhaus A, Kantarjian HM, Baccarani M, Lipton JH, Apperley JF, Druker BJ, Facon T, Goldberg SL, Cervantes F, Niederwieser D, Silver RT, Stone RM, Hughes TP, Muller MC, Ezzeddine R, Countouriotis AM, Shah NP: Dasatinib induces notable hematologic and cytogenetic responses in chronic-phase chronic myeloid leukemia after failure of imatinib therapy. Blood 2007;109:23032309.

10 Sherbenou DW, Hantschel O, Turaga L, Kaupe I, Willis S, Bumm T, Press RD, Superti-Furga G, Druker BJ, Deininger MW: Characterization of BCR-ABL deletion mutants from patients with chronic myeloid leukemia. Leukemia 2008;22:1184-1190.

11 Ruibao R: Mechanisms of BCR-ABL in the pathogenesis of chronic myelogenous leukemia. Nat Rev Cancer 2005;5:172-183.

12 Gruber FX, Hjorth-Hansen H, Mikkola I, Stenke L, Johansen T: A novel Bcr-Abl splice isoform is associated with the L248V mutation in CML patients with acquired resistance to imatinib. Leukemia 2006;20:20572060.
13 Borrow J: Guidelines for Mutation Analysis of BCR/ABL Kinase Domain: Interpreting TKI-Resistance Mutations in CML Patients. Birmingham, West Midlands Regional Genetics Laboratory, 2007.

14 Laudadio J, Deininger MW, Mauro MJ, Druker BJ, Press RD: An intron-derived insertion/truncation mutation in the BCRABL kinase domain in chronic myeloid leukemia patients undergoing kinase inhibitor therapy. J Mol Diagn 2008;10:177-180.

15 Azam M, Latek RR, Daley GQ: Mechanisms of autoinhibition and STI-571/imatinib resistance revealed by mutagenesis of $B C R$ ABL. Cell 2003;112:831-843.

16 Branford S, Rudzki Z, Walsh S, Parkinson I, Grigg A, Szer J, Taylor K, Herrmann R, Seymour JF, Arthur C, Joske D, Lynch K, Hughes T: Detection of BCR-ABL mutations in patients with CML treated with imatinib is virtually always accompanied by clinical resistance, and mutations in the ATP phosphate-binding loop (P-loop) are associated with a poor prognosis. Blood 2003;102:276283.

17 Hochhaus A, Kreil S, Corbin A, La Rosée P, Lahaye T, Berger U, Cross NCP, Linkesch W, Druker BJ, Hehlmann R: Roots of clinical resistance to STI-571 cancer therapy. Science 2001;293:2163a.

18 von Bubnoff N, Schneller F, Peschel C, Duyster J: $B C R-A B L$ gene mutations in relation to clinical resistance of Philadelphia-chromosome-positive leukaemia to STI571: a prospective study. Lancet 2002;359:487-491.

19 Nicolini FE, Corm S, Lê QH, Sorel N, Hayette S, Bories D, Leguay T, Roy L, Giraudier S, Tulliez M, Facon T, Mahon FX, Cayuela JM, Rousselot P, Michallet M, Preudhomme C, Guilhot F, Roche-Lestienne C: Mutation status and clinical outcome of 89 imatinib mesylate-resistant chronic myelogenous leukemia patients: a retrospective analysis from the French intergroup of CML (Fi(Phi)-LMC Group). Leukemia 2006;20:1061-1066.
20 Dai Z, Kerzic P, Schroeder WG, McNiece IK: Deletion of the Src homology 3 domain and $\mathrm{C}$-terminal proline-rich sequences in Bcr$\mathrm{Abl}$ prevents $\mathrm{Abl}$ interactor 2 degradation and spontaneous cell migration and impairs leukemogenesis. J Biol Chem 2001;276: 28954-28960.

-21 Wertheim JA, Perera SA, Hammer DA, Ruibao R, Boettiger D, Pear WS: Localization of $\mathrm{BCR}-\mathrm{ABL}$ to $\mathrm{F}$-actin regulates cell adhesion but does not attenuate CML development. Blood 2003; 102:2220-2228.

22 Fan P-D, Cong F, Goff SP: Homo- and hetero-oligomerization of the $\mathrm{c}-\mathrm{Abl}$ kinase and Abelson-interactor-1. Cancer Res 2003;63: 873-877.

23 Azam M, Nardi V, Shakespeare WC, Metcalf CA III, Bohacek RS, Wang Y, Sundaramoorthi R, Sliz P, Veach DR, Bornmann WG, Clarkson B, Dalgarno DC, Sawyer TK, Daley GQ: Activity of dual SRC-ABL inhibitors highlights the role of $\mathrm{BCR} / \mathrm{ABL}$ kinase dynamics in drug resistance. Proc Natl Acad Sci USA 2006; 103:9244-9249.

-24 Alvarez RH, Kantarjian HM, Cortes JE: The role of Src in solid and hematologic malignancies: development of new-generation Src inhibitors. Cancer 2006;107:1918-1929.

25 Khorashad JS, Lipton JH, Marin D, Milojkovic D, Cross NCP, Dibb N, Melo JV, Kamel-Reid S, Goldman JM, Apperley JF, Kaeda JS: Abnormally small BCR-ABL transcripts in CML patients before and during imatinib treatment (abstract 2153). Blood 2006;108:611a.

26 Volpe G, Cignetti A, Panuzzo C, Kuka M, Vitaggio K, Brancaccio M, Perrone G, Rinaldi M, Prato G, Fava M, Geuna M, Pautasso M, Casnici C, Signori E, Tonon G, Tarone G, Marelli O, Fazio VM, Saglio G: Alternative BCR/ABL splice variants in Philadelphia chromosome-positive leukemias result in novel tumor-specific fusion proteins that may represent potential targets for immunotherapy approaches. Cancer Res 2007;67: 5300-5307. 